\title{
X-RAY HALOES AND COOLING FLOWS
}

\author{
A.C. FABIAN \\ Institute of Astronomy, Madingley Road, \\ Cambridge CB3 0HA, UK
}

ABSTRACT. The properties of hot gaseous haloes in massive early-type galaxies are briefly reviewed. Gas flows in such haloes are complex yet so large-scale that they may guide us in the understanding of flows around disk galaxies. The intracluster medium is discussed as a further illustration of the properties of diffuse hot gas trapped in a gravtational well. Finally, the possibility of the existence of a significant diffuse medium in the Local Group, and in groups in general, is revived. Such a medium would generate a substantial disk-halo interaction with our Galaxy.

\section{INTRODUCTION}

Hot gaseous haloes are common in massive early-type galaxies, in groups and in clusters of galaxies. They provide an observable guide to what any large gaseous haloes of disk galaxies may be like and in particular show the behaviour of diffuse hot gas trapped in a gravitational potential. X-ray, and other, observations of the hot gas in early-type galaxies are reviewed and models for the flows in these galaxies discussed. The gas is shown to multiphase and the flows complex. Some clues for understanding the operation of thermal conduction, magnetic fields, mixing and star formation in such gas are considered. The more extensive, and better observed, hot atmospheres in clusters are used to illustrate these processes further.

Much of the gas in a protogalaxy may exist in the form of a hot halo if gravitational collapse succeeds in heating it to the virial temperature of the galaxy. The study of such haloes may therefore be important for understanding galaxy formation. Large-scale gaseous haloes may persist now in all groups of galaxies, as well as in clusters. This leads to disk-halo interactions being common, in the sense that the gas in disk galaxies in groups (i.e. perhaps most disk galaxies) is interacting with an intra-group or -cluster medium. Such interactions, which are commonly ignored in groups, may explain some observed phenomena in our own and other galaxies.

Dramatic disk-halo interactions occur when a group merges with another group or with a cluster of galaxies. Then the pressure of the surrounding halo rises tento onehundred-fold and the cluster halo controls the behaviour of the disk gas. 


\section{THE HOT INTERSTELLAR MEDIUM OF EARLY-TYPE GALAXIES}

The hot $\left(T \sim 10^{7} \mathrm{~K}\right)$ interstellar medium (ISM) of massive early-type galaxies was discovered by X-ray images from the Einstein Observatory (Forman et al. 1979). Prior to then, the apparent lack of any substantial ISM in elliptical galaxies was ascribed to galactic winds.

The X-ray data showed that several Virgo cluster ellipticals have substantial haloes of $10^{9}-10^{10} \mathrm{M}_{\odot}$ of hot gas. Of particular importance was the discovery of a 'plume' of X-ray emission to the galaxy M86, the radial velocity of which indicates it is falling through the intracluster medium (ICM) at about $1500 \mathrm{~km} \mathrm{~s}^{-1}$. A lack of radio, or other, emission in the plume indicated that the $\mathrm{X}$-ray emission was due to thermal bremsstrahlung and line radiation from diffuse hot gas. What $\mathrm{X}$-ray spectral data there was, agreed with that conclusion. The plume is then due to ram-pressure stripping of the hot halo of M86 by the Virgo cluster ICM.

These results were added to by observations of haloes in galaxies in groups (Biermann \& Kronberg 1983) and in relatively isolated ellipticals (Nulsen et al. 1984). Forman, Jones \& Tucker (1985), and Canizares, Fabbiano \& Trinchieri (1987) (for a review see Fabbiano 1989) have since scoured the Einstein Observatory database for detections and upper limits of the $\mathrm{X}$-ray luminosity of early-type galaxies and find that $L_{X} \propto L_{B}^{\beta}$, where $L_{X}$ and $L_{B}$ are the $\mathrm{X}$-ray and B-band luminosities of the galaxy and $\beta \sim 1.5-2.3$. The exact value obtained for $\beta$ depends on what corrections are made for other sources of X-ray emission (e.g. binaries) in the galaxy and on what distances are adopted (redshifts are not the best guide to distance in the nearby, Virgo distance, flow where most of the detected galaxies lie). The most recent determinations (see e.g. Sarazin 1990) prefer a value of $\beta \sim 2.3$. Ellipticals in clusters with a massive ICM, such as the Coma cluster, are deficient in such individual haloes (Canizares 1988), presumably due to ram-pressure stripping.

No clear detections have yet been made of X-ray emitting gaseous haloes around disk galaxies (Bregman \& Glassgold 1982; McCammon \& Sanders 1984). Indeed, disks and extensive hot haloes appear to be mutually exclusive, at least in the Einstein Observatory data, since Bender et al. (1989) have shown that X-ray haloes are most commonly found around 'boxy' galaxies and rarely around 'disky' ones (the terms 'boxy' and 'disky' refer to the shape of the isophotes). Whether this is due to an absence of gas around disk-, or 'disky'-galaxies, or to a lower temperature putting the emission outside the Einstein Observatory waveband is not clear. The recently launched X-ray telescope on ROSAT has much more sensitivity at softer $\mathrm{X}$-ray wavelengths and will resolve this issue, as well as finding hundreds more $\mathrm{X}$-ray emitting elliptical galaxies.

The detection of such haloes, particularly in isolated galaxies, indicated that the gas is quasi-static - any flows must be subsonic. If they were not, then the gas would disperse in a flow time,

$$
t_{\text {flow }}<R / c_{s}
$$


where $R$ is a characteristic radius for the bulk of the observed gas (say $10 \mathrm{kpc}$ ) and $c_{s}$ is the speed of sound in the gas $\left(\sim 10^{2} \mathrm{~km} \mathrm{~s}^{-1}\right.$, comparable with the velocity dispersion of stars within the galaxy). The mass loss rate of the galaxy would then be

$$
\dot{M} \gtrsim\left(\frac{M_{\text {gas }}}{t_{\text {flow }}}\right)>10 \mathrm{M}_{\odot} \mathrm{yr}^{-1} \text {. }
$$

This is much greater than the rate at which the ISM of the galaxy can be replaced by stellar mass loss,

$$
\dot{M}_{\star} \sim 1 \mathrm{M}_{\odot} \mathrm{yr}^{-1} .
$$

It would therefore be very surprising to detect haloes commonly in such galaxies. The simplest conclusion from the observed hot gaseous haloes is that the halo gas is in hydrostatic equilibrium.

This led to several conclusions which are somewhat controversial. The first is that the high temperature of the gas would lead to a wind developing and a loss of the gas unless the potential well of the galaxy was large. Massive elliptical galaxies have massive dark haloes (Forman, Jones \& Tucker 1975; Fabian et al. 1986; but see Trinchieri, Fabbiano \& Canizares 1986). The second is that the supernova rate in elliptical galxies must be low, less than one per $400 \mathrm{yr}$, or too much heat would be injected into the halo and it would be heated to the temperature required for escape. The supernova rate in ellipticals has been revised downward in the past few years (Van den Bergh, McClure \& Evans 1987) towards this value. One question that had worried me about supernovae in elliticals was the possible requirement of binaries if the supernovae were due to an old stellar population. Are binaries present in elliptical galaxies? This question in now answered to some degree by the discovery of novae in the radio galaxy Centaurus A, which does have a large spheroidal body of stars.

The third conclusion concerns the cooling rate of the hot halo. The radiative cooling time (due to the emission of X-rays such as those detected), $t_{c o o l}$ is less than a Hubble time within the inner $30 \mathrm{kpc}$ of many large ellipticals and is much shorter $\left(<10^{9} \mathrm{yr}\right)$ within the inner few kpc. This suggests that cooling flows operate in massive elliptical galaxies, where

$$
\dot{M}_{\text {cool }} \sim 1 \mathrm{M}_{\odot} \mathrm{yr}^{-1} \sim \dot{M}_{\star} .
$$

The accumulation of stellar mass loss in the hot halo is balanced by radiative cooling of the gas, presumably leading to some star formation. Whether 'normal' (disk-galaxy like) star formation continues to take place in early-type galaxies is debatable (Pickles 1986; Burstein et al.1988). Perhaps the gas chiefly forms lowmass stars, as must be the case in cluster cooling flows (see next Section).

Detailed studies of the X-ray surface brightness profile and of the effects of cooling and of mass and energy injection from stars and supernovae show that gas flows in ellipticals are complex (Thomas 1986; Thomas et al.1986; Sarazin \& White 1988; Sarazin \& Ashe 1989; Sarazin 1990). The gas must exist in many phases, some of which may flow out as some flow in. Gas cools out of the hotter phases 
over a wide range of radii, not just in the centre. Not all the stellar mass loss (predominantly from red-giant winds and planetary nebulae) may be heated to the $\mathrm{X}$-ray emitting phases, but the denser parts may just be slowed down and remain intact. What happens then is unclear. The flows probably resemble a giant galactic fountain, such as discussed elsewhere in this Volume for disk galaxies, except that this time the geometry is more neary spherical. Further study of such behaviour probably requires further X-ray observations of higher spatial and spectral quality. Fortunately these should soon be forthcoming from ROSAT, ASTRO-D and later X-ray missions in the 1990s.

The evolution of such haloes has been discussed by Mathews (1989; see also references in Sarazin 1990).

\section{THE INTRACLUSTER MEDIUM}

The ultimate hot halo is of course the intracluster medium (ICM). All clusters of galaxies appear to have diffuse gas spread throughout them with a mass of about 10 to 30 per cent of the total mass of the cluster. The gas has a temperature of between $2 \times 10^{7} \mathrm{~K}$ for the poorest clusters to $10^{8} \mathrm{~K}$ or more for the richest ones. Their X-ray properties have been well-reviewed by Sarazin (1988).

Such gas is not or particular relevance to the topic of the 'disk-halo connection', except that it may have stripped the disk gas from many member galaxies and its large scale means that some processes that must occur in hot halo gas have been clearly exposed for observation.

On the first point, it should be noted that if our galaxy could be placed in the core of a rich cluster, then the mean pressure of the ISM would rise by a factor of about 100. If the galaxy then fell in the gravitational well of the cluster it would experience large ram-pressure forces, first discussed by Gunn \& Gott in 1972, and turbulent stripping process (Nulsen 1982). Such a 'disk-halo' interaction would destroy the disk gas, and so its reason for being thin. This could account for some S0 galaxies (Biermann \& Shapiro 1979). Worth noting now is that clusters do not appear to have formed at some early time intact, but are evolving now through the merger of subclusters (Edge et al.1990; see also Gioia et al.1990). This means that whole groups are being subsumed into clusters now.

On the second point, we now see that the ICM is again complex, particularly in most cluster cores where the data are best. The radiative cooling time of the gas within about $100 \mathrm{kpc}$ of the central galaxy in most ( $\gtrsim 70$ per cent; Pesce et al. 1990) clusters is less than a Hubble time. The strongly-peaked X-ray surface brightness profiles within this region show that the cooling time continues to decrease inward, indicating the presence of a cooling flow. This just means that when the gas loses energy by radiation, its density must rise by inflow in order to maintain the pressure necessary to support the weight of the overlying gas. X-ray spectra of a few clusters shows that the gas does cool (Canizares, Markert \& Donahue 1988; Mushotzky \& Szymkowiak 1988). Instead of the one or less solar masses per year cooling in elliptical galaxies, here we have tens to hundreds of solar masses cooling out of 
the hotter phases per year. Optical colours and spectra of the central galaxies show that 'normal' star formation cannot be taking place and that whatever the cooled gas forms, it cannot be massive blue stars. Probably the gas just forms into low-mass stars, since the molecular clouds that give birth to massive stars in the Solar Neighbourhood cannot exist tens of $\mathrm{kpc}$ out from the central galaxy in the ICM. If one were to be placed there, it would be broken into pieces by the ICM on falling into the galaxy. Reviews of cooling flows and of how star formation might take place in them are given by Fabian, Nulsen \& Canizares (1984; 1990), Sarazin (1988) and Fabian (1990).

Points of interest for the study of hot haloes elsewhere are the operation of thermal instability (Balbus \& Soker 1989); the inference of turbulence in the gas (Heckman et al. 1989; Loewenstein \& Fabian 1990); of tangled magnetic fields which reduce thermal conduction (see above reviews - the magnetic fields themselves are studied on scales of kpc by Faraday depolarization of central radio sources; Dreher et al.1987; Fomalont et al.1989; Owen et al.1990); the occurrence of dust in the central galaxies, either in dust lanes such as in NGC 4696 (Sparks et al.1989) or observed through infrared emission (Bregman et al.1990), of warm clouds of gas embedded in the hot gas (see e.g. Heckman et al.1989), of cold HI clouds observed through $21 \mathrm{~cm}$ absorption of a radio source (Jaffe et al.1988; McNamara et al.1990), or $21 \mathrm{~cm}$ emission (Bregman et al.1988); of molecular gas observed through its CO emission (Lazareff et al.1989; Mirabel et al.1990). Jaffe's (1990) observation of extended HI absorption across the inner tens of kpc of the cooling flow in the Perseus cluster suggests that there is at least $10^{10} M_{\odot}$ of cold gas accumulated there. This could be a large underestimate of the total mass of cold gas in the flow if it is optically thick and extends (in clouds) out to radii of 100 - $200 \mathrm{kpc}$. We have been studying (Fabian et al.1990) the implications of such large quantities of cold gas. Cloud-cloud collisions may be common in the inner parts of the flow and contribute to the observed optical line emission. Cold clouds distributed throughout the core of the ICM may be responsible for some of the narrow absorption line clouds observed in the spectra of distant quasars. Further studies of the ICM should allow us to understand how magnetic fields, turbulence, cloud and star formation take place in all hot haloes.

\section{GAS FLOWS IN LOOSE GROUPS AND SPIRAL GALAXIES}

The general existence of hot haloes in early-type galaxies and clusters of galaxies suggests that they could exist in groups of galaxies. After all, it is unlikely that galaxy formation was 100 per cent efficient anywhere. The most difficult aspect is possibly the depth of the potential well in a loose group, such as the Local Group. The early star-forming phases of most galaxies are likely to be rich in supernovae (otherwise galaxies would not be metal-rich). These can blast much of the rest of the gas out of the potential well of small galaxies and lead to a metal-enriched intergalactic medium. This is probably the source of the metals in the ICM (Larson \& Dinerstein 1975; see discussion in Thomas \& Fabian 1990). A 
problem with a loose group is that much of the metal-enriched gas may be ejected out altogether. However, if the velocity is less than $100 \mathrm{~km} \mathrm{~s}^{-1}$, the gas can travel no more than $1 \mathrm{Mpc}$ in a Hubble time so will remain in the Group, if not bound to it. Consequently, we can expect that most groups, no matter how loose, contain an IntraGroup Medium, which is almost as metal-rich as that in clusters (say onetenth Solar). The temperature of this gas will be between $10^{6} \mathrm{~K}$ and $10^{7} \mathrm{~K}$ if it does not all form a wind, and the total mass of gas may even exceed that of the component galaxies in the group (the scaling arguments in Thomas \& Fabian 1990 are relevant here).

What limits can we place on the existence of such gas in the Local Group? For that we can use the soft X-ray Background limits of McCammon \& Sanders (1990), which have not changed significantly since the 1970 s, when Hunt \& Sciama (1972) made a similar estimate. Assuming a scale size of $1 \mathrm{Mpc}$, slightly larger than the distance to the Andromeda galaxy, I find a gas density of $\sim 10^{-5} \mathrm{~cm}^{-3}$ if $T \sim 10^{7} \mathrm{~K}$ and $2 \times 10^{-5} \mathrm{~cm}^{-3}$ if the temperature is about $3 \times 10^{6} \mathrm{~K}$. The total $\mathrm{X}$-ray luminosity is between $10^{41}-10^{42} \mathrm{erg} \mathrm{s}^{-1}$ and the mass of gas about $10^{12} \mathrm{M}_{\odot}$. The pressure of the gas is only a few percent of that in our own ISM, so there are no overpressure problems. The cooling time of the gas, if smoothly distributed, is between 1 and 100 Hubble times, so if the gas is clumpy, then cooling and cooled clouds may occur. The ram power due to the motion of our Galaxy through this medium is a few per cent of the total kinetic power due to supernovae. This is not much power compared with the total Galactic power budget, but the ram power is deposited at the outskirts of the galaxy, principally as turbulence (see Begelman \& Fabian 1990 for a discussion of a turbulent mixing layer above the galactic disk). Cold clouds embedded in the intragroup medium (perhaps clouds that have never been heated up, or regions that were denser and have since cooled) then impact our galaxy from a particular direction, related to the vector of our velocity and that of the local intragroup medium. They are then a source of high-velocity clouds.

Some discussions of the behaviour of such a medium can be found in the 1970s (Kahn \& Woltjer 1959; Hunt \& Sciama 1972; Oort 1969) and its effect on the Magellanic Stream (Mathewson et al.1977). It appears to me to have been largely ignored in the 1980s, except as a source for high-velocity clouds (see e.g. Mirabel \& Morras 1990), but does require further study, either to rule it out or to establish its properties. Simple ways in which such gas may be detected directly or indirectly are through its X-ray emission (either in the Local Group or in other groups ROSAT may be crucial here), through absorption studies of 'intragroup clouds' (perhaps some of the narrow metal absorption lines commonly found in the spectra of distant quasars, Bergeron (1988), are due to intragroup clouds) its impact on our and other galaxies - either in terms of energy deposited, mixing, turbulence, abrupt edges to the HI (see discussion in terms of photoionization for such edges by Kenney 1990) or HI warps, tails and streams. Some medium with roughly the properties described above must exist. 


\section{REFERENCES}

Balbus, S. \& Soker, N., 1989. Astrophys. J., 341, 611.

Begelman, M.C. \& Fabian, A.C., 1990. Mon. Not. R. astr. Soc., 244, 26 P.

Bender, R., Surma, P., Döbereiner, S., Möllenhoff, C. \& Madejsky, R., 1989. Astr. Astrophys., 217, 35.

Bergeron, J. 1988. In QSO Absorption Systems; Probing the Univers, eds Blades, J.C., Norman, C.A. \& Turnshek, D., C.U.P.

Biermann, P. \& Shapiro, S.L, 1979. Astrophys. J., 230, L33.

Biermann, P. \& Kronberg, P.P., 1983. Astrophys. J., 268, L69.

Bregman, J.N. \& Glassgold, A.E., 1982. Astrophys. J., 263, 564.

Bregman, J.D., McNamara, B. \& O'Connell, R., 1990. Astrophys. J., in press.

Bregman, J.N., Roberts, M.S. \& Giovanelli, R., 1988. Astrophys. J., 330, L93.

Burstein, D., Bertola, F., Buson, L.M., Faber, S.M. \& Lauer, T.R., 1988. Astrophys. J., $328,440$.

Canizares, C.R., 1988. In Cooling Flows in Clusters and Galaxies, ed. A.C. Fabian, Kluwer, Dordrecht, 376.

Canizares, C.R., Markert, T.H. \& Donahue, M.E., 1988. In Cooling Flows in Clusters and Galaxies, ed. A.C.Fabian, Kluwer, 63.

Canizares, C.R., Fabbiano, G. \& Trinchieri, G., 1987. Astrophys. J., 312, 503.

Dreher, J.W., Carilli, C.L. \& Perley, R.A., 1987. Astrophys. J., 316, 611.

Edge, A.C., Stewart, G.C., Fabian, A.C. \& Arnaud, K.A., 1989. Mon. Not. R. astr. Soc., 245, 559.

Fabbiano, G., 1989. Ann. Rev. Astr. Astrophys., 27, 87.

Fabian, A.C., 1990. In Baryonic Dark Matter, eds. D. Lynden Bell \& G. Gilmore, Kluwer, Dordrecht, 195.

Fabian, A.C., Nulsen, P.E.J. \& Canizares, C.R., 1984. Nature, 311, 733.

Fabian, A.C., Nulsen, P.E.J. \& Canizares, C.R., 1990. Astr. Astrophys. Rev., in press.

Fabian, A.C., Thomas, P.A., Fall, S.M. \& White, R.A., 1986b. Mon. Not. R. astr. Soc., $221,1049$.

Fabian, A.C., Thomas, P.A., Daines, S.J. \& Johnstone R.M., 1990. Preprint.

Fomalont, E.B., Ebnetter, K.A., van Breugel, W.J.M. \& Ekers, R.D., 1989. Astrophys. J., 346, L17.

Forman, W., Schwarz, J., Jones, C., Liller, W. \& Fabian, A., 1979. Astrophys. J., 234, L27.

Forman, W., Jones, C. \& Tucker, W., 1985. Astrophys. J., 293, 102.

Gioia, I.M., Henry, J.P., Maccacaro, T., Morris, S.L., Stocke, J.T. \& Wolter, A., 1990. Astrophys. J., 356, L35.

Gunn, J.E. \& Gott, J.R., 1972. Astrophys. J., 176, 1.

Heckman, T.M., Baum, S.A., van Breugel, W.J.M. \& McCarthy, P.,1989. Astrophys. J., $338,48$.

Hunt, R. \& Sciama, D.W., 1972. Mon. Not. R. astr. Soc., 157, 335.

Jaffe, W., de Bruyn, A.G. \& Sijbreng, D., 1988. In: Cooling Flows in Clusters and Galaxies, ed. Fabian, A. C., Kluwer, Dordrecht, Holland, p145.

Jaffe, W., 1990. Astr. Astrophys., in press.

Kahn, F.D. \& Woltjer, L., 1959. Astrophys. J., 130, 705.

Kenney, J.D.P., 1990. In The Interstellar Medium in External Galaxies, eds H.A. Thronson \& J.M. Shull, Kluwer, Dordrecht, 151.

Larson, R.B. \& Dinerstein, H.L., 1975. Publ. astr. Soc. Pacific, 87, 911.

Lazareff, B., Castets, A., Kim, D-W. \& Jura, M., 1989, 336, L13.

Loewenstein, M. \& Fabian, A.C., 1990. Mon. Not. R. astr. Soc., 242, 120. 
Mathews, W., 1989. Astr. J., 97, 42.

Mathewson, D.S., Schwarz, M.P. \& Murray, J.D., 1977. Astrophys. J., 217, L5.

McCammon, D. \& Sanders, W.T., 1990. Astrophys. J., 287, 167.

McCammon, D. \& Sanders, W.T., 1990. Ann. Rev. Astr. Astrophys., 28, 657.

McNamara, B.R., Bregman, J.N. \& O'Connell, R.W., 1990. Astrophys. J., 360, 20.

Mirabel, I.F. \& Morras, R., 1990. Astrophys. J., 356, 130.

Mirabel, I.F., Sanders, D.B. \& Kazes, I., 1989. Astrophys. J., 340, L9.

Mushotzky, R.F. \& Szymkowiak, A.E., 1987. In Cooling Flows in Clusters and Galaxies, ed. A.C.Fabian, Kluwer, 47.

Nulsen, P.E.J., 1982. Mon. Not. R. astr. Soc., 198, 1007.

Nulsen, P.E.J., Stewart, G.C. \& Fabian, A.C., 1984. Mon. Not. R. astr. Soc., 208, 185.

Oort, J.H., 1969. Nature, 224, 1158.

Owen, F., Eilek, J.A. \& Keel, W.C., 1990. Astrophys. J., 362, 449.

Pesce, J.E., Edge, A.C., Fabian, A.C. \& Johnstone, R.M., 1990. Mon. Not. R. astr. Soc., 244, 58.

Pickles, A.J., 1985. Astrophys. J., 296, 340.

Sarazin, C.L., 1988. X-ray Emission from Clusters of Galaxies, C.U.P.

Sarazin, C.L., 1990. In The Interstellar Medium in External Galaxies, eds H.A. Thronson \& J.M. Shull, Kluwer, Dordrecht, 201.

Sarazin, C.L. \& White, R.E., 1988. Astrophys. J., 335, 688.

Sarazin, C.L. \& Ashe, G.A., 1989. Astrophys. J., 345, 22.

Sparks, W.B., Macchetto, F. \& Golombek, D., 1989. Astrophys. J., 345, 153.

Thomas, P.A., 1986. Mon. Not. R. astr. Soc., 220, 949.

Thomas, P.A., Fabian, A.C., Arnaud, K.A., Forman, W. \& Jones, C., 1986. Mon. Not. R. astr. Soc., $222,655$.

Thomas, P.A. \& Fabian, A.C., 1990. Mon. Not. R. astr. Soc., 246, 156.

Trinchieri, G., Fabbiano, G. \& Canizares, C.R., 1986. Astrophys. J., 310, 637.

Van den Bergh, S., McClure, R.D. \& Evans, R., 1987. Astrophys. J., 323, 44.

Vedder, P.W., Trester, J.J. \& Canizares, C.R., 1988. Astrophys. J., 332, 725. 